\title{
Update Mammakarzinom 2019 Teil 2 - Implementierung neuer Diagnostika und Therapien bei Patientinnen mit fortgeschrittenem Brustkrebs in der klinischen Praxis
}

\author{
Update Breast Cancer 2019 Part 2 - Implementation \\ of Novel Diagnostics and Therapeutics in Advanced \\ Breast Cancer Patients in Clinical Practice
}

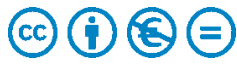

Autoren

Wolfgang Janni ${ }^{1}$, Andreas Schneeweiss ${ }^{2}$, Volkmar Müller ${ }^{3}$, Achim Wöckel ${ }^{4}$, Michael P. Lux ${ }^{5}$, Andreas D. Hartkopf ${ }^{6}$, Naiba Nabieva ${ }^{5}$, Florin-Andrei Taran ${ }^{6}$, Hans Tesch ${ }^{7}$, Friedrich Overkamp ${ }^{8}$, Diana Lüftner ${ }^{9}$, Erik Belleville ${ }^{10}$, Florian Schütz ${ }^{11}$, Peter A. Fasching ${ }^{5}$, Tanja N. Fehm ${ }^{12}$, Hans-Christian Kolberg ${ }^{13}$, Johannes Ettl ${ }^{14}$

Institute

1 Department of Gynecology and Obstetrics, Ulm University Hospital, Ulm

2 National Center for Tumor Diseases, Division Gynecologic Oncology, University Hospital Heidelberg, Heidelberg

3 Department of Gynecology, Hamburg-Eppendorf University Medical Center, Hamburg

4 Department of Gynecology and Obstetrics, University Hospital Würzburg, Würzburg

5 Erlangen University Hospital, Department of Gynecology and Obstetrics, Comprehensive Cancer Center ErlangenEMN, Friedrich-Alexander University Erlangen-Nuremberg, Erlangen

6 Department of Obstetrics and Gynecology, University of Tübingen, Tübingen

7 Oncology Practice at Bethanien Hospital Frankfurt, Frankfurt

8 OncoConsult Hamburg GmbH, Hamburg

9 Charité University Hospital, Campus Benjamin Franklin, Department of Hematology, Oncology and Tumour Immunology, Berlin

10 ClinSol GmbH \& Co KG, Würzburg

11 Department of Obstetrics and Gynecology, University of Heidelberg, Heidelberg

12 Department of Gynecology and Obstetrics, University Hospital Düsseldorf, Düsseldorf

13 Department of Gynecology and Obstetrics, Marienhospital Bottrop, Bottrop

14 Department of Obstetrics and Gynecology, Klinikum rechts der Isar, Technical University of Munich, Munich

Schlüsselwörter

Mammakarzinom, Metastasen, fortgeschritten, PI3K, PD-L1, Alpelisib, Atezolizumab, PARP, Supportivtherapie
Key words

breast cancer, metastatic, advanced, PI3K, PD-L1, alpelisib, atezolizumab, PARP, supportive therapy

eingereicht 13.1.2019

akzeptiert 28.1.2019

Bibliografie

DOI https://doi.org/10.1055/a-0842-6661

Geburtsh Frauenheilk 2019; 79: 268-281 @ Georg Thieme

Verlag KG Stuttgart · New York | ISSN 0016-5751

Korrespondenzadresse

Peter A. Fasching, MD

Erlangen University Hospital, Department of Gynecology and

Obstetrics, Comprehensive Cancer Center Erlangen EMN,

Friedrich Alexander University of Erlangen-Nuremberg

Universitätsstraße 21-23, 91054 Erlangen

peter.fasching@uk-erlangen.de

\section{ZUSAMMENFASSUNG}

Die Behandlung von Patientinnen mit fortgeschrittenem Mammakarzinom hat sich in den letzten Jahren weiterentwickelt. Zusätzlich zum Therapiefortschritt in den etablierten Subgruppen (Hormonrezeptor- und HER2-Status) gibt es nun Therapien, die sich an einzelnen molekularen Charakteristika orientieren, wie zum Beispiel die PARP-Inhibitortherapie bei BRCA-mutierten Patientinnen. Zusätzlich dazu sind Tests in der Entwicklung, die innerhalb von Subgruppen weitere Marker etablieren sollen, um die Wirksamkeit einer Therapie vorherzusagen. Die PI3K-Mutationstestung bei HER2-negativen, hormonrezeptorpositiven Tumoren, und die PD-L1-Testung von Immunzellen bei triple-negativen Tumoren werden voraussichtlich in der klinischen Praxis etabliert, um Patientinnen für die jeweiligen Therapien auszuwählen. Mit neuen The- 
rapieansätzen treten auch neue Nebenwirkungen auf. Das Management dieser Nebenwirkungen ebenso wie die der klassischen Therapien (supportive Therapie) ist mit der Einführung neuer Behandlungen essenziell, um die Lebensqualität der Patientinnen zu erhalten. Das Wissen über Maßnahmen zur Erhaltung und Verbesserung der Lebensqualität hat in den letzten Jahren deutlich zugenommen. Lifestyle-Faktoren sollten dabei ebenso Berücksichtigung finden wie moderne Therapieverfahren. Diese Übersichtsarbeit fasst die neuesten Studien und Veröffentlichungen zusammen und bewertet sie in Bezug auf die Relevanz für die klinische Praxis.

\section{ABSTRACT}

The treatment of patients with advanced breast cancer has developed further in recent years. In addition to therapeutic progress in the established subgroups (hormone receptor and HER2 status), there are now therapies which are geared to individual molecular characteristics, such as PARP inhibitor therapy in BRCA-mutated patients. In addition to this, tests are being developed which are intended to establish additional markers within subgroups in order to predict the efficacy of a therapy. PI3K mutation testing in HER2-negative, hormonereceptor-positive tumours and PD-L1 testing of immune cells in triple-negative tumours are expected to become established in clinical practice in order to select patients for the respective therapies. With new therapeutic approaches, new adverse effects also appear. The management of these adverse effects, just as those of classical therapy (supportive therapy), is essential with the introduction of new treatments in order to preserve patients' quality of life. Knowledge regarding measures to preserve and improve quality of life has significantly increased in recent years. Lifestyle factors should be taken into account, as should modern therapeutic methods. This review summarises the latest studies and publications and evaluates them in regard to the relevance for clinical practice.

\section{Einführung}

Im Zeitalter der Individualisierung von Therapien [1 - 4] erreichen aktuell weitere zielgerichtete und immunonkologische Substanzen bei definierten Patientinnengruppen mit fortgeschrittenem Mammakarzinom Zulassungsreife. Der Einsatz von CDK4/6-Inhibitoren bei HER2-negativen und hormonrezeptorpositiven fortgeschrittenen Mammakarzinompatientinnen ist Standard. Die Zulassung der PARP-Inhibitoren bei BRCA-mutierten Patientinnen steht unmittelbar bevor. Neue Studien zur Wirksamkeit von PI3KInhibitoren bei PI3K-mutierten Tumoren wurden ebenso vorgestellt wie Studien zur spezifischen Wirksamkeit von Checkpoint-Inhibitoren. Somit scheint die seit vielen Jahren angestrebte Individualisierung der Therapie in der klinischen Praxis angekommen zu sein [5]. Die folgende Übersichtsarbeit stellt die neuesten Studien und Veröffentlichungen aus diesem Kontext vor.

\section{Therapie des metastasierten triple-negativen Mammakarzinoms}

Die metastasierte Patientin mit einem triple-negativen Mammakarzinom (TNBC) bleibt weiterhin die große Herausforderung in der Onkologie. Der klassische therapeutische Ansatz ist die Chemotherapie. Allerdings ist diese Option mit einer kurzen progressionsfreien Zeit (PFS) verbunden; in der Second Line beträgt das PFS lediglich 9 Wochen und nur 49\% der Patientinnen erreichen die 3. Linie [6]. Das Gesamtüberleben liegt nach Diagnose der Metastasierung bei ca. 13-14 Monaten.

Während früher die Therapie analog zum nicht-triple-negativen Mammakarzinom erfolgte, empfiehlt die Kommission Mamma der AGO e. V. aktuell für diese Gruppe den Einsatz von platinhaltigen Therapien [7]. Zukünftige Ansätze differenzieren das TNBC möglicherweise in einen hochproliferierenden Subtyp, welcher weiterhin einer Chemotherapie bedarf, einen Subtyp mit nachweisbarer Androgenrezeptorexpression (mögliche antian- drogene Therapie), einen BRCA-assoziierten Typ (Einsatz von PARP-Inhibitoren) und einen immunassoziierten Subtyp, der durch Immuninfiltrate gekennzeichnet ist und den Einsatz von Checkpoint-Inhibitoren vielversprechend erscheinen lässt [8]. Nachdem mehrere Phase-II-Studien den Proof-of-Concept erbrachten, wurden kürzlich die Ergebnisse der IMpassion130-Studie veröffentlicht [8]. Im Rahmen dieser verblindeten Phase-IIIStudie wurden jeweils 451 Patientinnen mit einem nicht vortherapierten metastasierten TNBC 1:1 zu Nab-Paclitaxel $100 \mathrm{mg} / \mathrm{m}^{2}$, d1, 8, 15, q28d, oder der Kombination von Nab-Paclitaxel und dem PD-L1-Inhibitor Atezolizumab randomisiert. Neben der vorherigen taxanhaltigen Therapie und dem Vorliegen von Lebermetastasen war der PD-L1-Status auf den Immunzellen (IC, positiv bei $\geq 1 \%$ ) wichtigster Stratifizierungsfaktor. In der Intention-totreat-(ITT-)Analyse lag das PFS nach einem medianen Follow-up von 12,9 Monaten im experimentellen Arm bei 7,2 vs. 5,5 Monaten im Standardarm (HR 0,80 [95\%-KI 0,69-0,92]; $\mathrm{p}=0,0025$ ). Das Gesamtüberleben erreichte mit einer HR von 0,83 (95\%-KI 0,69-1,02) und einem Unterschied von 21,3 zu 17,6 Monaten noch keine statistische Signifikanz $(p=0,0840)$. Es wurde, wenn auch vom statistischen Plan noch nicht vorgesehen, das Gesamtüberleben (OS) der Patientinnen mit einem PD-L1-IC-positiven Tumor deskriptiv betrachtet. Hierbei zeigte sich für diese Subgruppe ein bedeutsamer Unterschied von 15,5 zu 25,0 Monaten (HR 0,62 [95\%-KI 0,45-0,86]) - für dieses herausfordernde Kollektiv ein vielversprechendes und Practice-changing-Ergebnis, falls sich der Überlebensvorteil in der endgültigen Analyse bestätigen sollte. Dennoch blieben viele Fragen offen, die jüngst bei der Präsentation weiterer Subgruppenanalysen beantwortet wurden [9]. In Bezug auf den PD-L1-Status basierte die Rationale, die Positivität der umgebenden Immunzellen zu berücksichtigen, auf der Tatsache, dass triple-negative Tumorzellen selten eine PD-L1-Expression aufweisen; in der vorliegenden Studie nur 9\% der Tumorzellen vs. $41 \%$ bei Berücksichtigung der Immunzellen. Dass der PD-L1-IC-Status essenziell für das Ansprechen ist, bestätigte sich durch die negativen Ergebnisse der PD-L1-IC-negativen Subgrup- 
pe - das PFS lag sowohl im Standard- als auch experimentellen Arm bei 5,6 Monaten $(p=0,5152)$ und das OS bei 18,9 vs. 18,4 Monaten $(p=0,9068)$. Weitere Biomarker wie die CD8-Positivität oder das Vorliegen von stromalen tumorinfiltrierenden Lymphozyten (TiLs) waren nur prädiktiv für einen klinischen Benefit, wenn auch eine PD-L1-IC-Positivität vorlag. Mit der kommenden Einführung der PARP-Inhibitoren stellt sich zudem die Frage, in wie weit Atezolizumab auch bei Vorliegen einer BRCA-Mutation wirksam ist. Bei Vorliegen einer BRCA-Mutation war die Effektivität nur durch den PD-L1-Status definiert. PD-L1-IC-negative Mutationsträgerinnen hatten sowohl für PFS und OS keinen Vorteil durch Atezolizumab, während für PD-L1-IC-positive Mutationsträgerinnen insbesondere für das PFS ein Benefit nachgewiesen werden konnte (HR 0,45 [95\%-KI 0,21-0,96]; $P=0,04$ ). Dementsprechend könnte sich die Kombinationstherapie mit dem Checkpoint-Inhibitor für die Subgruppe der PD-L1-IC-positiven Patientinnen unabhängig von weiteren Biomarkern in Kürze zum neuen Standard in der First-Line-Situation entwickeln.

Die Situation zeigt, dass die molekulare Diagnostik bei Patientinnen mit metastasiertem Mammakarzinom zunehmen wird. Zum einen wird bei den triple-negativen Mammakarzinomen eine Testung der Immunzellen für PD-L1 notwendig werden. In der Impassion 130 -Studie waren ca. $40 \%$ der Patientinnen positiv für diesen Biomarker, genauso wie eine Testung für BRCA1 und BRCA2 bei HER2-negativen Mammakarzinomen. In triple-negativen Mammakarzinomen konnte hier eine Mutation in 10-20\% der Fälle gefunden werden [10-15]. Therapiesequenzen sind noch nicht etabliert, jedoch spricht die Verlängerung des OS für eine primäre Therapie mit dem PD-L1-Antikörper.

\section{Therapie des metastasierten hormonrezeptor- positiven HER2-negativen Mammakarzinoms}

In den letzten 10 Jahren sind einige Substanzen getestet und teilweise auch mit großem Erfolg in die Klinik eingeführt worden, die bei einer Kombination mit einer antihormonellen Therapie bei einem Teil der Patientinnen die endokrine Resistenz überwinden oder die Wirksamkeit der Antihormontherapie verbessern und so zu einem längeren PFS führen. Nach der Einführung von Everolimus $[16,17]$ konnte in 7 Studien gezeigt werden, dass die CDK4/ 6-Inhibitoren (Palbociclib, Ribociclib und Abemaciclib) bei präund postmenopausalen Patientinnen und in mehreren Therapielinien das PFS verlängern mit Hazard Ratios zwischen 0,5 und 0,6 (zusammengefasst in [18]). Zusätzlich zu einem verbesserten PFS wurde von einer der Studien auch ein Trend für ein besseres OS berichtet [19]. Ebenso konnte gezeigt werden, dass die Lebensqualität durch die Progressionsverzögerung verbessert werden konnte $[20,21]$.

Da sich schon 1 Jahr nach der Verfügbarkeit die Therapie mit einem CDK4/6-Inhibitor in der 1. Therapielinie als Standard etabliert hat [22], stellt sich die Frage, wie diese Patientinnen bei Abbruch der CDK4/6-Inhibitortherapie weiterbehandelt werden. Trotz der Empfehlung der Ausschöpfung der antiendokrinen Therapie wurde bei einem großen Teil der Patientinnen eine Sequenz von mehreren Chemotherapien eingesetzt [23]. Dieses könnte durch weitere, wirksame Kombinationstherapien zusätzlich ver- bessert werden in Richtung einer häufigeren antiendokrinen Therapie zusätzlich verbessert werden. Circa $40 \%$ der hormonrezeptorpositiven HER2-negativen Mammakarzinome haben Mutationen im PI3K-Gen, was die häufigste genetische Aberration bei diesem Tumortyp ist [24,25]. Die Mutationen können zu Tumorzellwachstum und endokriner Resistenz führen. Für eine Kombinationstherapie mit CDK4/6-Inhibitoren wurde des Weiteren beschrieben, dass sich Mutationen, z. B. die PI3K-Mutation, in mehr als $8 \%$ der Patientinnen neu anreichern [26]. Deswegen wäre eine Therapie mit einem PI3K-Inhibitor durchaus sinnvoll. Vor Kurzem wurden die Daten einer prospektiv randomisierten Phase-III-Studie (SOLAR-1) zum PI3K-Inhibitor Alpelisib vorgestellt [27, 28]. Alpelisib ist ein spezifischer Inhibitor der PIK3CA-Isoform und hemmt speziell die mutierte Untereinheit. In der SOLAR-1-Studie wurden 572 Patientinnen mit fortgeschrittenem hormonrezeptorpositiven, HER2-negativen Mammakarzinom in 2 Kohorten aufgeteilt, jene mit und ohne eine PIK3CA-Mutation. Die Mehrzahl der Patientinnen war postmenopausal, und alle hatten eine Vortherapie mit einem Aromatasehemmer und ca. $10 \%$ mit einem CDK4/6-Inhibitor erhalten. Die Mutationsanalyse erfolgte im Primärgewebe [27] für die primäre Analyse und in einer sekundären retrospektiven Untersuchung in zirkulierender Tumor-DNA im Plasma (liquid biopsy) [28].

Die Patientinnen wurden in beiden Kohorten in 2 Behandlungsarme randomisiert: Fulvestrant plus Alpelisib vs. Fulvestrant plus Placebo. Etwa die Hälfte der Patientinnen hatte viszerale Metastasen. Die Studie erreichte ihren primären Endpunkt: Die Kombination von Alpelisib + Fulvestrant velängerte das PFS der PIK3CAmutierten Patientinnen von 5,7 auf 11 Monate gegenüber dem Kontrollarm (HR 0,65; 95\%-KI: 0,50-0,85; p=0,00065). Dagegen zeigte sich kein signifikanter Vorteil für die Kombinationstherapie in der nicht mutierten Kohorte. Die Subgruppenanalyse zeigte für die PIK3CA-mutierten Patientinnen einen konsistenten Vorteil für die Kombinationstherapie. Zum Zeitpunkt der 1. Interimsanalyse waren die Ergebnisse im Gesamtüberleben dagegen noch nicht reif.

Insgesamt war die Kombinationstherapie im Vergleich zu andern bislang untersuchten PI3K-Inhibitoren relativ gut verträglich. Hauptnebenwirkung war eine Hyperglykämie mit ca. 35\% (Grad 3-4) sowie Hauttoxizitäten (Rash) mit einer Häufigkeit von $10 \%$ (Grad 3-4). Ungefähr zwei Drittel der mutierten Patientinnen benötigten eine Therapieunterbrechung oder Dosisreduktion unter der Kombination, und 25\% brachen die Therapie vorzeitig ab. Die Ergebnisse dieser Biomarker-getriggerten Studie sollten zur Zulassung führen. Allerdings sind die Nebenwirkungen für die Patientinnen belastend und erfordern ein besonderes Management. Ebenso könnte die Implementierung der PI3K-Mutationstestung eine Herausforderung darstellen. Hierbei muss bedacht werden, dass die Testung sowohl an in Paraffin eingebettetem Tumormaterial als auch an ctDNA im Blut stattfinden kann. Die Analyse, bei der die Patientinnen nach einer Mutation, die an ctDNA nachgewiesen wurde, betrachtet wurden, zeigte mit einer Hazard Ratio von 0,55 eine ähnliche Wirksamkeit [28]. 
Keimbahn-Genetik von FCGR
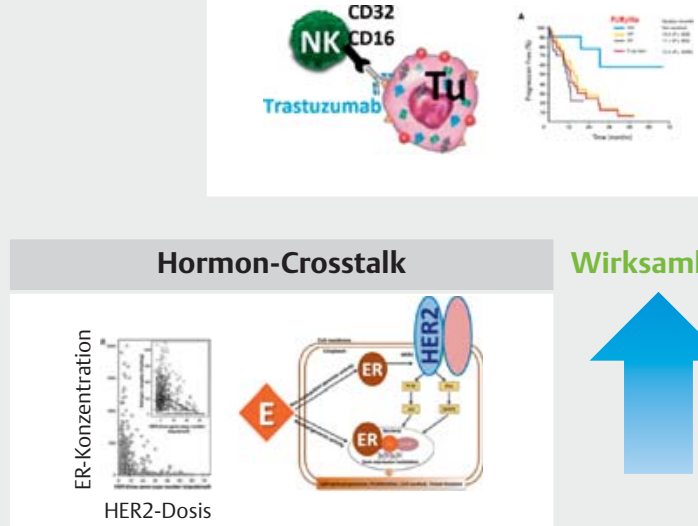

Hormon-Crosstalk

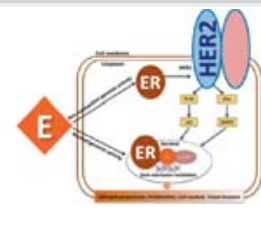

Wirksamkeit

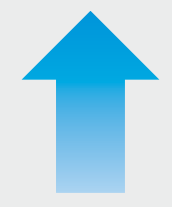

Prognose

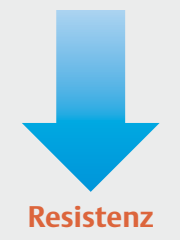

somatische HER2-Mutationen

aktivierende HER2- mit schlechterer Prognose Mutationen bei assoziierte HER2-Mutationen vielen Krebsentitäten
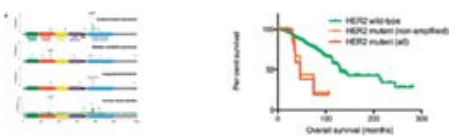
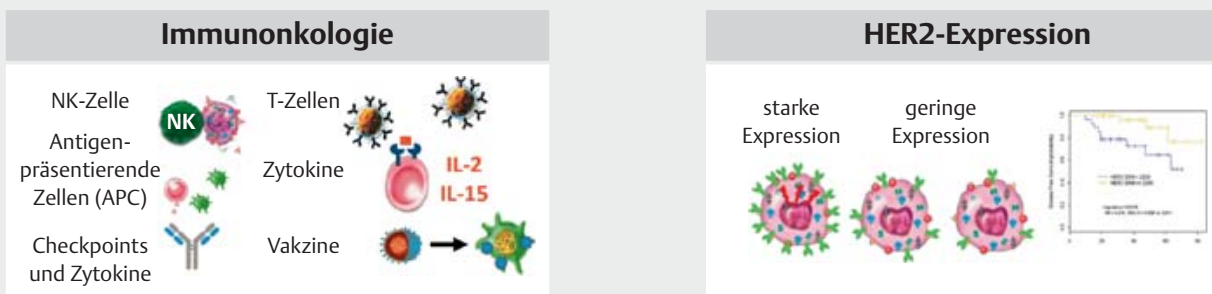

- Abb. 1 Referenzmechanismen bei einer Anti-HER2-Therapie (NK: Natural-Killer-Zelle, E: Östrogen, ER: Östrogenrezeptor; modifiziert nach: [32-37]).

\section{Therapie des metastasierten HER2-positiven Mammakarzinoms}

Die Entdeckung der Amplifikation von HER2 mit einer damit assoziierten ungünstigen Prognose [29] und die nachfolgende Entwicklung des Antikörpers Trastuzumab $[30,31]$ haben die Behandlung von Patientinnen mit HER2-positivem, fortgeschrittenem Mammakarzinom in den letzten nunmehr fast 20 Jahren deutlich verändert. Die Einführung der Substanzen Pertuzumab und Trastuzumab Emtansin (T-DM1) konnten einige der Resistenzmechanismen [32-37] überwinden ( $\bullet \mathbf{A b b}$. 1) und eine deutliche Verlängerung des PFS und auch des OS erreichen [3841], was zur Implementierung in nationale und internationale Leitlinien geführt hat [42]. Eine kürzlich veröffentlichte Arbeit des PRAEGNANT-Netzwerks [43] zeigt die Einführung dieser Therapien in die „real-world“ klinische Praxis [44]. Diese Arbeit konnte zeigen, dass über $80 \%$ aller HER2-positiven Patientinnen bis zur 4. Therapielinie Trastuzumab erhalten hatten, ca. 70\% die Kombination von Trastuzumab und Pertuzumab, ca. 50\% Lapatinib und ebenfalls ca. 50\% T-DM1 [44]. Die Sequenz von Trastuzumab + Pertuzumab gefolgt von T-DM1 wurde bis zur 4. Therapielinie ca. $40 \%$ der Patientinnen gegeben. Patientinnen mit einem negativen Hormonrezeptorstatus oder einem hohen Grading schienen diese Sequenz häufiger erhalten zu haben [44].

Mit dem Antikörper-Drug-Konjugat (ADC) T-DM1 ist eine wirksame, neuartig wirkende Therapie eingeführt worden. DS-8201a ist ein weiteres ADC, welches derzeit in klinischen Studien getestet wird. Das chemotherapeutische Agens (DXd, ein Topoisomerase-I-Inhibitor) ist mit einem Linker an Trastuzumab gebunden, welcher das Chemotherapeutikum nach Bindung an das HER2Molekül freisetzt [45, 46]. Dem Molekül wird zugeschrieben, auch bei Patientinnen mit niedriger HER2-Expression eine Wirksamkeit zu haben. Dies konnte auch in einer sehr großen Phase-I-Studie gezeigt werden [47]. Allerdings kam es bei knapp über 240 behandelten Patientinnen zu einem mit einer Pneumonitis assoziierten Todesfall. Die Aufarbeitung der Fälle kommt zur Schlussfolgerung, dass eine Therapie bei Kontrolle dieser ernstzunehmenden Nebenwirkung mit einem intensiven Monitoring, der Beendigung der Medikation mit DS-8201a bei Auftreten und einer Behandlung mit Kortikosteroiden möglich ist [48]. Momentan wird diese Substanz nach T-DM1 in einer Phase-II-Studie bei Patientinnen mit HER2-Überexpression getestet (NCT03523585).

Die Therapiesequenz Pertuzumab + Trastuzumab $\rightarrow$ T-DM1 wird zwar von den Leitlinien unterstützt, jedoch sind Pertuzumab und T-DM1 parallel in klinischen Studien entwickelt worden, sodass an der EMILIA-Studie keine mit Pertuzumab vorbehandelten Patientinnen teilgenommen hatten [41]. Das mediane PFS war 9,6 Monate [41]. Daten zum medianen PFS sind nun ebenfalls aus dem PRAEGNANT-Netzwerk veröffentlicht worden. Für Patientinnen, die nach Pertuzumab T-DM1 erhalten hatten, waren die PFS-Zeiten 7,7, 4,2 und 4,0 Monate für Patientinnen in der 2., 3. und 4. Therapielinie [49]. Allerdings muss angemerkt werden, dass die Fallzahl mit 57 Patientinnen klein war. 


\section{Die besondere, metastasierte Situation - Hirnmetastasen}

Die Verbesserung der Behandlungsoptionen von Patientinnen mit metastasiertem Mammakarzinom hat zu einem verlängerten Überleben geführt. Bei verbesserter Kontrolle der Metastasierung entwickeln nunmehr rund 30\% aller metastasierten Patientinnen im Laufe ihrer Erkrankung Hirnmetastasen. Hirnmetastasen stellen oft den limitierenden Faktor der Erkrankung dar, da das Überleben nach Auftreten einer Hirnmetastasierung meist nur wenige Monate beträgt. Neben der schlechten Prognose kommt es durch kognitive und neurologische Defizite zu einer massiven Einschränkung der Lebensqualität. Patientinnen mit HER2-positiven oder triple-negativen Karzinomen entwickeln häufiger Hirnmetastasen. Eine kürzlich vorgestellte Untersuchung hat sich mit der Inzidenz zerebraler Metastasen als ersten Ort einer Metastasierung nach adjuvanter Therapie bei Patientinnen mit HER2-positivem Mammakarzinom beschäftigt. Hierzu wurde das Follow-up der Patientinnen aus der BCIRG-006-Studie ausgewertet, die den Einsatz von Trastuzumab in der adjuvanten Therapie des Mammakarzinoms untersucht hat [30]. Von den 3222 Patientinnen haben $17,8 \%$ bei einer medianen Nachbeobachtungszeit von 10,3 Jahren Fernmetastasen entwickelt. 17,5\% $(n=101)$ von diesen Fernmetastasen waren Hirnmetastasen als erste Lokalisation der Metastasierung. Es ließ sich kein Unterschied in der Häufigkeit mit und ohne Trastuzumab beobachten. Als Risikofaktoren für die Entwicklung zerebraler Metastasen konnten ein negativer Hormonrezeptorstatus und mehr als 3 befallene axilläre Lymphknoten identifiziert werden. Insgesamt ist allerdings noch sehr wenig zu Therapie und Prognose von Patientinnen mit Hirnmetastasen bekannt, die außerhalb klinischer Studien behandelt wurden. Um die Datenlage hierfür zu verbessern, wurde das Register „Brain Metastases in Breast Cancer (BMBC)“ zur Erfassung der deutschen Versorgungsrealität initiiert. Neben einer bereits publizierten Analyse zum Outcome von rund 1700 in Deutschland behandelten Patientinnen [50] wurde aktuell eine weitere Auswertung vorgestellt. Hierbei wurde ein bereits publizierter Prognosescore, das „breast-graded prognostic assessment (GPA)“ [51], in der deutschen Kohorte validiert [52]. Dieser Score basiert auf den Faktoren Karnofsky-Status, biologischer Subtyp des Tumors und Alter der Patientin. Die Abschätzung der Prognose von Patientinnen ist in der klinischen Routine beispielsweise für die Entscheidung über Radikalität therapeutischer Maßnahmen von Relevanz. Das mediane Überleben in den Subgruppen variierte in der deutschen Kohorte zwischen 2,4 und 12,3 Monaten. Der Score konnte hierbei gut zwischen verschiedenen Prognosegruppen differenzieren, allerdings war die Überlebenszeit kürzer als in der publizierten Kohorte von Sperduto et al., die zwischen 3,4 und 25,3 Monate betrug. Dies unterstreicht, dass es durchaus Unterschiede im absoluten Überleben in verschiedenen klinischen Kohorten geben kann. Noch ungünstiger als das Überleben von Patientinnen mit Hirnmetastasen ist die Prognose von Patientinnen mit einem Befall der Hirnhäute (Meningeosis carcinomatosa). Wie bei zerebralen Metastasen fehlen auch hier Daten zu Optionen einer systemischen Therapie. In einer kleinen Kohorte von 7 Patientinnen mit hormonrezeptorpositivem und HER2-negativem Mammakarzi- nom wurden nun Daten für die Effektivität des CDK4/6-Inhibitors Abemaciclib vorgestellt [53]. Es konnte ein Therapieansprechen bei einzelnen Patientinnen sowie ein Gesamtüberleben von 8,4 Monaten beobachtet werden, welches länger als in Vergleichskohorten ist. Die Studie wird aktuell fortgesetzt.

Zusammenfassend ist das Problem der zerebralen Metastasierung zunehmend Fokus von Forschungsanstrengungen, die hoffentlich in absehbarer Zeit zur Verbesserung der Behandlung beitragen werden.

\section{CTCs und DTCs als Biomarker beim Mammakarzinom}

Der prognostische Wert von disseminierten Tumorzellen (DTC) aus dem Knochenmark und zirkulierenden Tumorzellen (CTC) aus dem Blut wurde bereits in mehreren Arbeiten nachgewiesen [5461], wobei die Datenlage für CTCs ausgedehnter als für DTCs ist. Trotz allem sind disseminierte Tumorzellen von besonderer Relevanz. Kürzlich wurde gezeigt, dass im Rahmen der Karzinogenese von Brustkrebs einzelne Zellen schon sehr früh die primären Läsionen verlassen und verantwortlich für einen metastatischen Rückfall sind [62]. Im Rahmen einer großen gepoolten Analyse konnten Hartkopf et al. die prognostische Relevanz von DTCs an 10307 Patientinnen mit frühem Mammakarzinom bestätigen [63]. Bei 27,3\% aller Patientinnen wurden Tumorzellen im Knochenmark zum Zeitpunkt der Primärdiagnose nachgewiesen, und der Nachweis war mit einem signifikant schlechteren OS (HR: 1,23, $p=0,006$ ) bzw. krankheitsfreien Überleben (DFS) (HR: 1;30; $p<0,001)$ vergesellschaftet. Es zeigte sich zudem, dass vor allem DTC-positive Patientinnen mit Luminal-B-Tumoren (definiert als HR+/HER2-/G3) ein höheres Fernmetastasierungsrisiko haben (HR: 2,34). Ob die Bestimmung von DTCs als Prädiktionsmarker für adjuvante Therapiestrategien, z.B. eine Behandlung mit Bisphosphonaten, verwendet werden kann, wird derzeit im Rahmen prospektiver Studien untersucht (z. B. NCT01545648).

Bei Patientinnen mit metastasiertem Mammakarzinom ist der Nachweis von mindestens 5 zirkulierenden Tumorzellen (CTC) im peripheren Venenblut ein negativer prognostischer Faktor [64]. Bidard et al. stellten daher die Frage, ob der Nachweis von CTCS (mindestens 5 CTCs/7,5 ml Blut mittels CellSearch, Menarini Silicon Biosystems, Castel Maggiore BO, Italien) bei Patientinnen mit hormonrezeptorpositivem, HER2-negativem Mammakarzinom im fortgeschrittenen Stadium als Entscheidungskriterium herangezogen werden kann, ob eine endokrine Therapie (ET) ausreichend oder eine Chemotherapie mit anschließender endokriner Therapie (CTX) notwendig ist [65]. Hierfür wurden 761 Patientinnen in die prospektiv randomisierte Phase-III-STIC-CTC-Studie eingeschlossen. Es erfolgte eine 1:1-Randomisierung. Im Standardarm wurde nach Einschätzung des behandelnden Arztes (ET oder CTX) therapiert. Im CTC-Arm wurde bei < 5 CTCs eine ET und bei $\geq 5$ CTCs eine CTX eingesetzt. Primärer Endpunkt war der Vergleich des progressionsfreien Überlebens beider Arme. Hier war der CTC-Arm dem Kontrollarm nicht unterlegen. In einer geplanten Subgruppenanalyse zeigte sich zudem, dass Patientinnen mit $\geq 5$ CTCs auch dann von einer Chemotherapie profitieren, wenn von klinischer Seite eine endokrine Therapie als ausreichend ange- 
sehen wurde [65]. Die Autoren kommen daher zu dem Schluss, dass CTCs als Marker für die Anwendung einer Chemotherapie herangezogen werden könnten. Zwei Fragen bleiben allerdings offen. Zum einen sollte in weiteren Studien geklärt werden, wie die Studienergebnisse im Zeitalter der CDK4/6-Inhibition in die klinische Praxis integriert werden können. Zum anderen ist unklar, ob bei Patientinnen mit < 5 CTCs auf eine intensivere Behandlung verzichtet werden kann. Die Ergebnisse der STIC-CTC-Studie sind vor allem deshalb wichtig, weil erstmals prospektiv randomisiert gezeigt wurde, dass bestimmte Patientinnen mit hormonrezeptorpositivem, HER2-negativem fortgeschrittenem Mammakarzinom von einer Chemotherapie (zumindest im Vergleich zu einer rein endokrinen Behandlung) profitieren.

\section{Supportive Therapie}

Die supportive Therapie ist ein fundamentaler, aber auch komplexer Teil der onkologischen Therapie und Betreuung, der ein hohes Maß an interdisziplinärer Zusammenarbeit und ein vertrauensvolles Verhältnis zwischen Patient und Arzt fordert. Verschiedene Leitlinienkommissionen und Experten-Panels haben versucht, die Herausforderungen und die daraus resultierenden Empfehlungen zusammenzufassen [7,66-70]. Eine Übersicht über die Behandlungsfelder und notwendigen Überlegungen präsentiert $>$ Tab. 1. Für die meisten der relevanten, klinisch bedeutsamen Nebenwirkungen existieren Arbeitsgruppen, die Behandlungsschemata ausarbeiten [71 - 78]. Für den praktischen Gebrauch sind digitalisierte Applications wie in $\mathbf{A} \mathbf{A b} \mathbf{b} \mathbf{2}$ entwickelt worden. Während Studien für neue onkologische Therapien häufig mit vielen Ressourcen durchgeführt werden, entwickeln sich spezifische sup-

- Tab. 1 Beispiele für supportive Maßnahmen (modifiziert nach [7, 66 - 68, 70]).

\begin{tabular}{|c|c|c|}
\hline supportive Maßnahme & grundlegend & weiterführend \\
\hline Patienteninformation & $\begin{array}{l}\text { Informationen über Krankheit, Therapie, } \\
\text { Nebenwirkungen und Selbsthilfegruppen }\end{array}$ & Patienten- und Angehörigenveranstaltungen \\
\hline Angehörigeninformation & $\begin{array}{l}\text { Informationen über Krankheit, Therapie, } \\
\text { Nebenwirkungen und Selbsthilfegruppen }\end{array}$ & Patienten- und Angehörigenveranstaltungen \\
\hline Arztinformation & $\begin{array}{l}\text { kontinuierliche Information und Weiterbildung } \\
\text { über neue und komplexe Therapien }\end{array}$ & $\begin{array}{l}\text { Etablierung einer multidisziplinären Versorgung } \\
\text { von Nebenwirkungen für spezielle Therapien }\end{array}$ \\
\hline Psychoonkologie & Vorhalten einer psychoonkologischen Versorgung & Patienten- und Angehörigenveranstaltungen \\
\hline Seelsorge & Vorhalten einer Seelsorge & Patienten- und Angehörigenveranstaltungen \\
\hline muskulo-skeletale Maßnahmen & Beratung über körperliche, funktionelle Aktivität & physikalische Therapie, medikamentöse Therapie \\
\hline Ernährung und Verdauung & $\begin{array}{l}\text { bei einigen Therapien Peristaltikhemmer } \\
\text { (z. B. Loperamid), ggf. Infektionsdiagnostik }\end{array}$ & $\begin{array}{l}\text { bei Bedarf Peristaltikhemmer, antikonstipative } \\
\text { Therapie, Infektionsdiagnostik, Diätberatung }\end{array}$ \\
\hline Stomatitis & Beratung über Ernährung, Nahrungsaufnahme & topische Therapie \\
\hline Übelkeit und Erbrechen & $\begin{array}{l}\text { Antiemetika nach Leitlinie (inkl. Steroide, HT3-i } \\
\text { und NK1-i) }\end{array}$ & Verhaltenstherapie, psychoonkologischer Support \\
\hline Hautnebenwirkungen & Aufklärung & topische Therapie \\
\hline Infusionsschäden & Aufklärung & DMSO, Dexrazoxane, chirurgische Therapie \\
\hline Neurotoxizität & Aufklärung, Schmerztherapie, physikalische Therapie & medikamentöse Therapie \\
\hline Kardiotoxizität, Rhythmus & $\begin{array}{l}\text { Aufklärung und Kenntnis über kardiotoxische } \\
\text { Substanzen, Monitoring der kardialen Funktion } \\
\text { (LVEF, QTc-Zeit) }\end{array}$ & \\
\hline Myelosuppression & $\begin{array}{l}\text { Kontrolle der Blutwerte, Kenntnis über Therapien, } \\
\text { die eine primäre Prophylaxe fordern }\end{array}$ & $\begin{array}{l}\text { Colony Stimulating Factors, Erythropoetin, } \\
\text { Transfusionen }\end{array}$ \\
\hline Infektionen & Hepatitis-B-Screening, antiinfektiöse Therapie & Reservetherapeutika \\
\hline Fatigue & Aufklärung & psychosoziale Betreuung \\
\hline Schlafstörungen & Aufklärung & Verhaltenstherapie \\
\hline Schmerzen & Stufen-Schmerztherapie, physikalische Therapie & Schmerzspezialist \\
\hline Fertilität & $\begin{array}{l}\text { Verhütung unter Therapie, Effekte der Therapie } \\
\text { auf Fertilität }\end{array}$ & Kryokonservierung, medikamentöser Fertilitätserhalt \\
\hline menopausale Beschwerden & Aufklärung über Therapieeffekte & symptomatische Therapie \\
\hline Knochengesundheit & $\begin{array}{l}\text { Bisphosphonate, Denosumab, körperliche } \\
\text { Bewegung, Ernährungsberatung }\end{array}$ & \\
\hline Lifestyle & Beratung & \\
\hline Langezeitkomplikationen & Aufklärung, programmierte Nachsorge & symptomorientierte Therapie \\
\hline
\end{tabular}




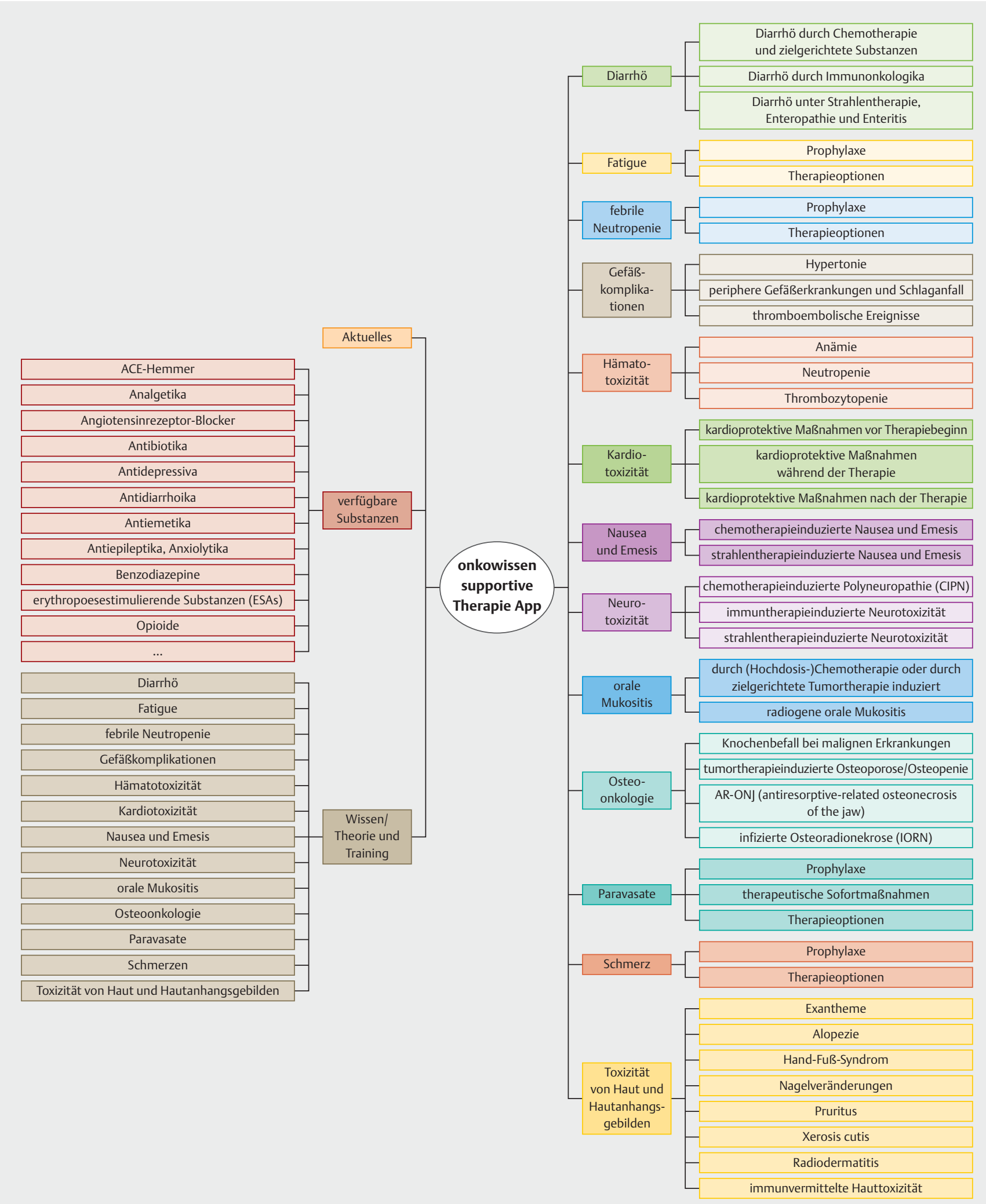

- Abb. 2 Supportivmaßnahmen wie in der Application von www.onkowissen.de beschrieben. 
portive Therapien erst danach und das Wissen über die Vermeidung von Kurz- und Langzeittoxizitäten folgt oft erst Jahre nach einem breiten Einsatz der Medikamente. Im Folgenden seien einige aktuelle Studien erwähnt.

\section{Anthrazyklinbedingte Kardiotoxizität: keine sichere medikamentöse Prävention}

Die Bedeutung der Kardiotoxizität einer anthrazyklinhaltigen Therapie wird oft erst in Langzeituntersuchungen deutlich. In einer 10-Jahres-Untersuchung beim Vergleich dreier Therapien (anthrazyklinhaltige Chemo [A] vs. A + Trastuzumab [T] vs. anthrazyklinfreie Chemo + Trastuzumab) konnten in der 10-Jahres-Nachbeobachtung 5-mal so viele kardiale Todesfälle im A+T-Arm gefunden werden wie im anthrazyklinfreien Arm [79]. Währen bei HER2-positiven Patientinnen die Option einer anthrazyklinfreien Therapie etabliert ist, ist die Datenlage beim HER2-negativen Mammakarzinom dünner, obwohl es Hinweise gibt, dass eine anthrazyklinfreie Therapie ähnlich wirksam ist [80]. Sind Patientinnen jedoch auf eine anthrazyklinhaltige Therapie angewiesen, stellt sich die Frage nach einer möglichen Prävention unter der Therapie. Kürzlich wurde zu diesem Thema eine der bisher größten Studien vorgestellt [81]. Es wurde untersucht, ob sich eine anthrazyklinbedingte Kardiotoxizität durch einen ACE-Hemmer oder einen $\beta$-Blocker reduzieren lässt. Untersucht wurden 468 Patientinnen mit primärem HER2-positivem Mammakarzinom, die eine adjuvante Therapie mit Trastuzumab erhielten. Die zuvor applizierte Chemotherapie konnte anthrazyklinbasiert oder anthrazyklinfrei sein. In der 3-armigen Studie wurden $\beta$-Blocker (Carvedilol) vs. ACE-Hemmer (Lisinopril) vs. Placebo untersucht. Als primäre Endpunkte wurden definiert: Abfall der Ejektionsfraktion (EF) um mind. 10\% oder um 5\% und ein Absinken unter den Grenzwert von < $50 \%$. Der Beobachtungszeitraum betrug 2 Jahre. Die Patientinnen wurden stratifiziert nach anthrazyklinhaltiger und anthrazyklinfreier Therapie.

In der anthrazyklinfreien Kohorte gab es keine Unterschiede. In der anthrazyklinhaltigen Kohorte zeigte sich, dass die Kardiotoxizität sowohl durch den ACE-Hemmer (37\%), als auch durch den $\beta$-Blocker (31\%) reduziert werden konnte. Aber auch im Placeboarm hatten $47 \%$ eine Kardiotoxizität.

Somit wurde durch die Studie zwar der potenzielle Einsatz von Kardiaka zur Kardioprotektion bestätigt, aber das Ergebnis der Studie ist mit Blick auf die Resultate im Kontrollarm unbefriedigend. Möglicherweise ist die EF als Surrogatmarker ein unzureichender Parameter für den primären Endpunkt. Die Definition der manifesten Herzinsuffizienz wäre präziser und aussagefähiger gewesen.

Die Tatsache, dass im anthrazyklinfreien Stratum keine reduzierte Kardiotoxizität beobachtet wurde, stärkt den Trend in Richtung anthrazyklinfreier Therapien.

\section{Hitzewallungen: Oxybutynin reduziert Intensität und Häufigkeit}

Hitzewallungen sind ein Thema, das nicht nur die Lebensqualität von Patientinnen beeinträchtigt, sondern unter Umständen auch einen Effekt auf die Prognose haben könnte. So ist bekannt, dass Frauen mit Nebenwirkungen eine schlechtere Compliance (Adhärenz) unter einer antihormonellen Therapie aufweisen [82,83].
Des Weiteren konnte nachgewiesen werden, dass eine reduzierte Compliance einen Einfluss auf die Prognose von postmenopausalen hormonrezeptorpositiven Patientinnen unter einer Aromatasehemmertherapie haben kann [84]. Eine supportive Medikation könnte deswegen in vielfacher Hinsicht bedeutsam sein, da eine hormonelle Substitution nicht indiziert ist. In einer randomisierten, doppelblinden, placebokontrollierten Studie wurde das Anticholinergikum Oxybutynin, das zur Behandlung einer Überaktivität der Harnblasenmuskulatur zugelassen ist, aber auch gegen Hitzewallungen wirksam erscheint, untersucht. Oxybutynin wurde in der 3-armigen Studie oral in 2 Dosierungen (2,5 mg und $5 \mathrm{mg}$ ) vs. Placebo getestet. Die hier verwendete Dosis ist erheblich niedriger als die meist in der Therapie einer überaktiven Blase verwendete. Eingeschlossen wurden Frauen mit einer hohen Frequenz von Hitzewallungen. Die meisten Frauen befanden sich unter einer Therapie mit Tamoxifen oder einem Aromatasehemmer. Die Behandlungsdauer betrug 6 Wochen nach einer Baseline-Woche ohne Medikation. Intensität und Frequenz der Hitzewallungen wurden über verschiedene Fragebögen evaluiert. Als primärer Endpunkt war die Veränderung in der wöchentlichen Intensität und Frequenz von Hitzewallungen definiert.

Sowohl die Intensität als auch die Häufigkeit von Hitzewallungen konnte signifikant gesenkt werden $(p<0,01)$, unabhängig von der applizierten Dosis von Oxybutynin. Die Nebenwirkungen waren akzeptabel und betrafen vor allem Mundtrockenheit, Harnverhalt, trockene Augen, Diarrhö und Kopfschmerzen. Beim Vergleich mit anderen gegen Hitzewallungen eingesetzten Substanzen wie z. B. Fluoxetin, Citalopram oder Venlafaxin schneidet Oxybutynin deutlich besser ab. Da die Studie insgesamt nur $150 \mathrm{~Pa}-$ tientinnen beobachtet hatte, bleibt abzuwarten, wie sich die Medikation in einer größeren Population verhält.

\section{Antiemese im breiten Einsatz}

Die Antiemese hat sich in den letzten Jahren zu einer hocheffektiven supportiven Therapie entwickelt. In der Ära vor der Einführung der 5-HT3-Rezeptor-Antagonisten wurde bei einer Standardchemotherapie für Mammakarzinompatientinnen in der Regel $\mathrm{Er}$ brechen in über $60 \%$ der Patientinnen berichtet [85]. Heutzutage sind diese Raten mithilfe einer Prophylaxe mit Steroiden, 5-HT3Rezeptoranagonisten und Neurokinin-1-(NK1-)Rezeptor-Antagonisten, wie in aktuellen Leitlinien empfohlen [75] und in einer großen Studie in der klinischen Routine beschrieben [86], deutlich niedriger. Eine Analyse von fast 1000 Mammakarzinompatientinnen ( $n=986)$, die eine Standardchemotherapie und das Kombinationspräparat NEPA (Netupitant + Palonosetron) erhalten haben, konnte zeigen, dass Erbrechen in nur ca. 10\% der Frauen und Übelkeit in ca. 30-40\% der Patientinnen [86] auftrat. Dies verdeutlicht die großen Fortschritte bei der supportiven Therapie dieser Nebenwirkung, die vor wenigen Jahrzehnten noch einen deutlichen Einfluss auf die Lebensqualität von Patientinnen unter Chemotherapie hatte.

Der Einsatz von Antiemetika wird zu einem großen Teil von dem emetogenen Risiko einer Chemotherapie abhängig gemacht. Hauptprädiktor ist hierbei die Art der Chemotherapie. Individuelle Faktoren können auch in Betracht gezogen werden. Individuelle molekulare Prädiktoren sind noch nicht etabliert, obwohl vielversprechende Ergebnisse bereits vorhanden sind [87- 


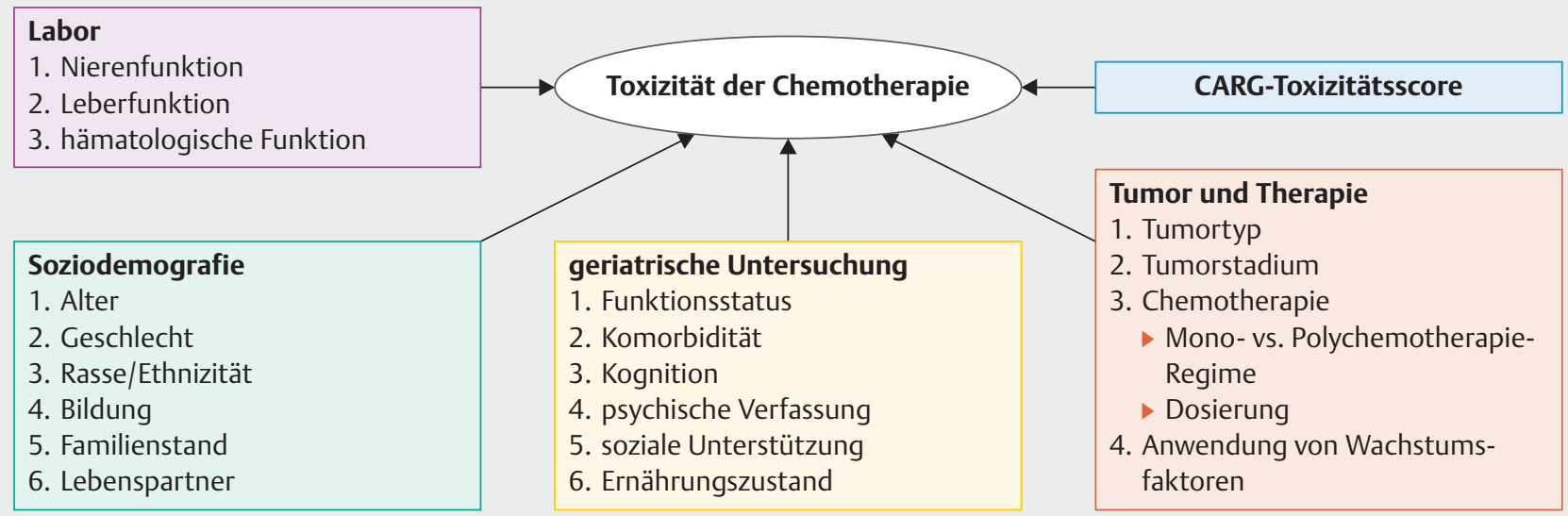

- Abb. 3 Zusammensetzung und Konzept des CARG (Cancer \& Aging Research Group) Toxicity Tools.

90]. Nächste Schritte könnten auch hier die Individualisierung der Therapie sein.

\section{Lifestyle zur Prävention, Verbesserung der Prognose und Unterstützung der Mammakarzinomtherapie}

Viele neue Ansätze haben das Thema „Lebensqualität“ im Auge. Dies bezieht sich auf das Befinden der Patientinnen unter verschiedenen Therapieformen, die mögliche Veränderung durch bestimmte Verhaltensregeln wie auch die Lebensqualitätsmessung. Während es für klinisch erfahrene OnkologInnen geradezu selbstverständlich ist, dass eine adjuvante Chemo- oder Antihormontherapie im Vergleich zu einer rein endokrinen Behandlung zu einer (zumindest passageren) Verschlechterung der Lebensqualität führt, fehlten hierfür bislang randomisierte Daten. Im Rahmen der TAILOR-X-Studie [91] wurden nun Lebensqualitätsdaten erhoben, welche an über 10000 Brustkrebspatientinnen insbesondere im Intervall von 3-6 Monaten nach Therapiebeginn eine Verschlechterung der kognitiven Leistung, Fatigue und endokriner Ausfallserscheinungen aufzeigen konnten [92]. Insofern ist es klinisch schlüssig, dass sich andere Gruppen aufmachten, dieses „tiefe Tal“ der Lebensqualität (und mit Einschränkungen der Prognose) unter adjuvanter Chemotherapie durch „Lifestyle“-Interventionen zu verbessern.

Klinisch praktikable Ergebnisse konnte in 2 kürzlich präsentierten Studien vor allem durch Training gezeigt werden [93, 94]. Die finnische Studiengruppe um die „EDDA“-Studien untersuchte, ob ein 12-monatiges Kraft- und Ausdauertraining unter adjuvanter Chemotherapie die kardiopulmonale Leistung, ausgewiesen durch die $\mathrm{VO}_{2}$ max, verbessern kann [94]. Das Trainingsprogramm war sehr anspruchsvoll mit insgesamt 4 Stunden Training pro Woche, davon 2 Stunden unter persönlicher Anleitung eines Physiotherapeuten; die Kontrollgruppe erhielt eine Beratung, wie nach norwegischen Standards üblich. Die Adhärenz lag bei $70 \%$ bei einem Beobachtungszeitraum von 1 Jahr. Die ausgeprägteste Ver- schlechterung der $\mathrm{VO}_{2}$ max fand sich bei Kontrollpatientinnen unter einer taxanhaltigen Chemotherapie mit einem durchschnittlichen Verlust um 17\% nach 6 Monaten und Persistenz der Minderung um 7,3\% nach 1 Jahr, während die beübten Patientinnen dann nur noch einen Verlust von 1,4\% erlebten.

Die deutsche SUCCESS-Studiengruppe hatte einen ähnlichen Ansatz gewählt, allerdings mittels telefonischer Beratungen und Postversendungen ohne persönlichen Trainerkontakt über die Gesamtdauer von 2 Jahren [93]. Auch war die Patientinnenselektion eine andere, da nur Patientinnen mit einem Body-Mass-Index (BMI) von $24-40 \mathrm{~kg} / \mathrm{m}^{2}$ aufgenommen wurden. Unter beratender Aufsicht verloren die Patientinnen innerhalb von 2 Jahren $1 \mathrm{~kg}$ an Körpergewicht, während die Kontrollpatientinnen $1 \mathrm{~kg}$ an Gewicht zunahmen. Allerdings lag in dieser Untersuchung die Adhärenz nur bei rund $50 \%$. Betrachtet man nur die sogenannten „Completer“, also über den Beobachtungszeitraum von 2 Jahren adhärente Patientinnen, so sieht man einen Unterschied von mehr als 3 kg Körpergewicht zwischen den beiden Armen und eine Hazard Ratio zugunsten der Interventionsgruppe von 0,51 in der multivariaten Analyse des krankheitsfreien Überlebens [93]. All diese Ergebnisse stützen die Notwendigkeit der Beratung sehr, denn der relative Effekt der Gewichtsreduktion und Beübung ist damit genauso relevant wie der Effekt einer adjuvanten chemotherapeutischen oder endokrinen Behandlung.

Nichtsdestotrotz verbleibt die schwere Aufgabe, die Therapiefähigkeit älterer Patientinnen richtig einzuschätzen. Hierfür wurde das CARG (Cancer \& Aging Research Group) Toxicity Tool entwickelt, das sich aus unterschiedlichen Parametern der Kategorien soziodemografische Angaben, Tumor- und Therapieeinzelheiten sowie Laborparametern zusammensetzt ( Score korreliert hervorragend mit den hochgradigen Toxizitäten und der Notwendigkeit der Dosisreduktion, Therapieverschiebung und Hospitalisation, und stellt damit eine hervorragende Hilfestellung bei der Entscheidung über die Durchführbarkeit einer adjuvanten Chemotherapie bei älteren Patientinnen dar. 


\section{Biomarker und genomische Charakterisierung}

Während die Etablierung molekularer Marker in der Vergangenheit eine lange Zeit gedauert hat [96], liegt die Herausforderung in der Zukunft darin, die Fülle der Biomarker in einen sinnvollen klinischen Kontext zu setzen. Ein Fokus beim metastasierten Mammakarzinom liegt in der Beschreibung der genomischen Charakterisierung und dem Nachweis spezieller Mutationen, die im Rahmen der Metastasierung oder der Progression auftreten bzw. diese mitbedingen. So ist bekannt, dass beispielsweise unter dem Druck einer endokrinen Therapie ESR1-Mutationen der Tumorzellen im Rahmen einer Metastasierung vermehrt auftreten können [26]. Bislang gibt es wenig Evidenz zu weiteren Mutationsmustern. Im Rahmen einer neueren Studie [97] wurde Tumormaterial und Blutserum von 629 Patientinnen mit metastasierter Erkrankung analysiert. Das Kollektiv stammte aus 6 französischen Studien (SAFIR-01 [98], SHIVA [99], MOSCATO [100], SAFIR-02 (NCT02299999), PERMED-01 (NCT02342158), MATCH-R (NCT02517892). Ziel war eine „Whole-Exome“-Sequenzierung des Tumorgewebes und der Serum-DNA (HiSeq: $n=262$ /Novaseq: $\mathrm{n}=367$ ) zur Identifizierung genomischer Muster und dem Vergleich zwischen frühem $(\mathrm{eBC})$ und metastasiertem (mBC) Mammakarzinom. Die Tumorbiologie der 629 mBC-Patientinnen zeigte folgende Verteilung: $n=387$ : HR+/HER2-, $n=186$ : TNBC, $n=32$ : HER2+. Biopsien wurden zum Großteil aus der Leber entnommen (272 Patientinnen =43,2\%), zu geringeren Anteilen aus Lymphknoten (111 Patientinnen $=17,6 \%$ ) oder anderen Metastasierungslokalisationen. Insgesamt zeigte sich eine hohe Heterogenität und klonale Diversität zwischen den Mutationsmustern, die im Rahmen einer Metastasierung in Relation zur frühen Erkrankung dramatisch zunehmen. Dies betraf jedoch vor allem HR+/HER2$\mathrm{mBC}$, während bei TNBC bereits im Rahmen der frühen Erkrankung eine höhere Diversität vorlag. Im Gesamtkollektiv zeigten sich vor allem 9 Treibermutationen (TP53, NF1, RB1, RBMX, FRG1, ESR1, RIC8A, AKT1, KRAS), die vermehrt bei mBC-Patientinnen in Relation zu eBC-Patientinnen auftraten und teilweise mit einem schlechteren Outcome assoziiert sind. Diese waren nachweisbar bei Patientinnen mit HR+/HER2- mBC, nicht jedoch bei HER2+ $\mathrm{mBC}$ oder mTNBC. Mutationen, die als therapeutische Targets zu sehen sind (PIC3CA, BRCA2) waren bei HR+/HER2- mBC signifikant häufiger als bei HER2+ mBC oder mTNBC nachweisbar. Drei Mutationssignaturen (S13 [APOBEC], S10 [POLE], S17 [ohne Namen]) wurden häufiger im Metastasengewebe nachgewiesen im Vergleich zum frühen Mammakarzinom. Diese gelten als Surrogat „genomischer Evolution“, und der Nachweis dieser Signaturen war ebenfalls mit einem schlechteren Outcome assoziiert, insbesondere, wenn diese in Kombination auftraten. Diese Signaturen waren ebenfalls bei HR+/HER2- mBC nachweisbar, während bei $\mathrm{mTNBC}$ aufgrund einer enormen Heterogenität keine Signaturen abzuleiten waren. Interessanterweise zeigte jedoch eine Untergruppe der mTNBC-Patientinnen somatische biallele „lossof-funktion-Mutationen“ (LoF) auf Genen, die für Hormonrezeptorkaskaden kodieren und damit möglicherweise eine Population für eine Therapie mit PARP-Inhibitoren darstellen könnten. Zieht man die Häufigkeit von Keimbahnmutationen von BRCA1 und
$B R C A 2$ in Betracht und ggf. auch weitere Homologous-Repair-Gene wie CHEK2, ATM, BARD1, PALB2 und RAD51D [10,11, 101, 102], so könnte der Gesamtanteil der Patientinnen, die für eine PARP-Inhibitortherapie infrage kommen, bei über 10\% aller Mammakarzinompatientinnen sein. Dies muss aber in Studien noch belegt werden.

Die Strukturierung der Einführung solcher multigenomischen Ansätze benötigt eine strukturierte, ggf. computerunterstützte Verwaltung. Der Support des Arztes durch Systeme, die möglicherweise durch maschinelles Lernen unterstützt werden, könnte ein Ansatz sein, diese Big-Data-Analysen in die klinische Praxis einzuführen $[103,104]$.

\section{Ausblick}

Auch wenn alle beschriebenen Studien und Ergebnisse einen großen wissenschaftlichen Nutzen haben, sind die direkten klinischen Herausforderungen übersichtlich. Die Implementierung der BRCA-Testung aller HER2-negativen fortgeschrittenen Mammakarzinompatientinnen, die PI3K-Mutationstestung der HER2-negativen, hormonrezeptorpositiven Patientinnen und das Therapiemanagement bei Anti-PD1/PD-L1-Therapien und Anti-PI3K-Therapien scheinen die Hauptaufgaben der nächsten Monate zu sein, um optimal auf die kommenden Therapien vorbereitet zu sein.

\section{Danksagung}

Diese Arbeit entstand teilweise in Folge von Förderungen der Firma Riemser und des PRAEGNANT-Netzwerks, das von den Firmen Hexal, Pfizer, Celgene, Daiichi-Sankyo, Roche, Merrimack, Eisai und Novartis unterstützt wird. Keine der Fimen hatte einen Anteil bei der Verfassung dieses Manuskriptes. Für den Inhalt des Manuskriptes sind alleine die Autoren verantwortlich.

Interessenkonflikt

A.D.H. hat Sprecher- und Beraterhonorare von AstraZeneca, Genomic Health, Roche, Novartis, Celgene, Lilly, MSD, Eisai, Teva, Tesaro, DaiichiSankyo, Hexal und Pfizer erhalten. N. N. hat Beraterhonorare von Janssen-Cilag und Novartis bezogen. F. O. hat Sprecher- und Beraterhonorare von Amgen, AstraZeneca, Bayer, BMS, Boehringer-Ingelheim, Chugai, Celgene, Cellex, Eisai, Gilead, Hexal, Ipsen, Janssen-Cilag, Merck, MSD, Novartis, Riemser, Roche, Tesaro und Teva erhalten. F.-A. T. hat Honorare von AstraZeneca, Genomic Health und Novartis erhalten. H.-C. K. hat Honorare von Carl Zeiss meditec, TEVA, Theraclion, Novartis, Amgen, AstraZeneca, Pfizer, Janssen-Cilag, GSK, LIV Pharma, Roche und Genomic Health bezogen. P. A. F. hat Honorare von Novartis, Pfizer, Roche, Amgen, Celgene, Daiichi-Sankyo, AstraZeneca, Merck-Sharp \& Dohme, Eisai, Puma und Teva erhalten. An seiner Einrichtung werden Forschungsarbeiten mit finanzieller Unterstützung von Novartis und Biontech durchgeführt. H. T. hat Honorare von Novartis, Roche, Celgene, Teva, Pfizer sowie Reisekostenzuschüsse von Roche, Celgene und Pfizer erhalten. J. E. hat Honorare von AstraZeneca, Roche, Celgene, Novartis, Lilly, Pfizer, Pierre Fabre, Teva sowie Reisekostenzuschüsse von Celgene, Pfizer, Teva und Pierre Fabre erhalten. M.P. L. war Mitglied von Beratungsgremien für AstraZeneca, MSD, Novartis, Pfizer, Eisai, Genomic Health und Roche und hat Vortragshonorare von MSD, Lilly, Roche, Novartis, Pfizer, Genomic Health, AstraZeneca, medac und Eisai bezogen. V.M. hat Sprecherhonorare von Amgen, AstraZeneca, Celgene, Daiichi-Sankyo, Eisai, Pfizer, Novartis, Roche, Teva, Janssen-Cilag sowie Beraterhonorare von Genomic Health, Hexal, Roche, Pierre Fabre, Amgen, Novartis, MSD, Daiichi-Sankyo und Eisai, Lilly, Tesaro und Nektar 
erhalten. E. B. hat Honorare von Novartis, Celgene, Riemser, Pfizer, Hexal, Amgen und onkowissen.de für Beratung sowie Tätigkeiten in den Bereichen Management von klinischer Forschung und medizinische Fortbildung erhalten. A. S. hat Honorare von Roche, Celgene, AstraZeneca, Novartis, Pfizer, Zuckschwerdt Verlag GmbH, Georg Thieme Verlag, Aurikamed $\mathrm{GmbH}, \mathrm{MCl}$ Deutschland $\mathrm{GmbH}$, bsh medical communications $\mathrm{GmbH}$ und promedicis $\mathrm{GmbH}$ bezogen. W. J. hat Honorare und Forschungsmittel von Novartis, Roche, Pfizer, Lilly, AstraZeneca, Chugai, Sanofi, Daichi und Tesaro erhalten. F. S. war Mitglied von Beratungsgremien für Novartis, Lilly, Amgen und Roche und hat Vortragshonorare von Roche, AstraZeneca, MSD, Novartis und Pfizer erhalten. A.W. war Mitglied von Beratungsgremien für Novartis, Lilly, Amgen, Pfizer, Roche, Tesaro, Eisai und hat Vortragshonorare von Novartis, Pfizer, Aurikamed, Roche, Celgene erhalten. D. L. hat Honorare von Amgen, AstraZeneca, Celgene, Lilly, Loreal, MSD, Novartis, Pfizer, Tesaro und Teva erhalten. T.N. F. war Mitglied von Beratungsgremien für Amgen, Daichi Sankyo, Novartis, Pfizer und Roche und hat Vortragshonorare von Amgen, Celgene, Daichi Sankyo, Roche, Novartis und Pfizer bezogen.

Literatur

[1] Taran FA, Schneeweiss A, Lux MP et al. Update Breast Cancer 2018 (Part 1) - Primary Breast Cancer and Biomarkers. Geburtsh Frauenheilk 2018; 78: $237-245$

[2] Schneeweiss A, Lux MP, Janni W et al. Update Breast Cancer 2018 (Part 2) - Advanced Breast Cancer, Quality of Life and Prevention. Geburtsh Frauenheilk 2018; 78: 246-259

[3] Wockel A, Lux MP, Janni W et al. Update Breast Cancer 2018 (Part 3) Genomics, Individualized Medicine and Immune Therapies - in the Middle of a New Era: Prevention and Treatment Strategies for Early Breast Cancer. Geburtsh Frauenheilk 2018; 78: 1110-1118

[4] Muller V, Wockel A, Lux MP et al. Update Breast Cancer 2018 (Part 4) Genomics, Individualized Medicine and Immune Therapies - in the Middle of a New Era: Treatment Strategies for Advanced Breast Cancer. Geburtsh Frauenheilk 2018; 78: 1119-1128

[5] Fasching PA, Schneeweiss A, Kolberg HC et al. Translational highlights in breast cancer research and treatment: recent developments with clinical impact. Curr Opin Obstet Gynecol 2019; 31: 67-75

[6] Kassam F, Enright K, Dent R et al. Survival outcomes for patients with metastatic triple-negative breast cancer: implications for clinical practice and trial design. Clin Breast Cancer 2009; 9: 29-33

[7] Kommission Mamma der Arbeitsgemeinschaft Gynäkologische Onkologie e.V. in der Deutschen Gesellschaft für Gynäkologie und Geburtshilfe e.V. sowie in der Deutschen Krebsgesellschaft e.V. Diagnostik und Therapie von Patientinnen mit primärem und metastasiertem Brustkrebs. 2018. Online: https://www.ago-online.de/de/infothek-fueraerzte/leitlinienempfehlungen/mamma/; Stand: 07.07.2018

[8] Schmid P, Adams S, Rugo HS et al. Atezolizumab and Nab-Paclitaxel in Advanced Triple-Negative Breast Cancer. N Engl J Med 2018; 379 : 2108-2121

[9] Emens LA, Loi S, Rugo HS et al. IMpassion130: Efficacy in immune biomarker subgroups from the global, randomized, double-blind, placebocontrolled, phase III study of atezolizumab + nab-paclitaxel in patients with treatment-nave, locally advanced or metastatic triple-negative breast cancer. San Antonio Breast Cancer Symposium 2018; Abstr. GS104

[10] Couch FJ, Hart SN, Sharma P et al. Inherited mutations in 17 breast cancer susceptibility genes among a large triple-negative breast cancer cohort unselected for family history of breast cancer. J Clin Oncol 2015; 33 : 304-311

[11] Fasching PA, Hu C, Hart SN et al. Cancer predisposition genes in metastatic breast cancer - Association with metastatic pattern, prognosis, patient and tumor characteristics [abstract]. In: Proceedings of the 2017 San Antonio Breast Cancer Symposium; 2017 Dec 5-9; San Antonio, TX Philadelphia (PA): AACR. Cancer Res 2018; 78: Abstr. PD1-02
[12] Fasching PA, Loibl S, Hu C et al. BRCA1/2 Mutations and Bevacizumab in the Neoadjuvant Treatment of Breast Cancer: Response and Prognosis Results in Patients With Triple-Negative Breast Cancer From the GeparQuinto Study. J Clin Oncol 2018. doi:10.1200/JCO.2017.77.2285

[13] Hahnen E, Lederer B, Hauke J et al. Germline Mutation Status, Pathological Complete Response, and Disease-Free Survival in Triple-Negative Breast Cancer: Secondary Analysis of the GeparSixto Randomized Clinical Trial. JAMA Oncol 2017; 3: 1378-1385

[14] Wunderle M, Gass P, Haberle L et al. BRCA mutations and their influence on pathological complete response and prognosis in a clinical cohort of neoadjuvantly treated breast cancer patients. Breast Cancer Res Treat 2018; 171: 85-94

[15] Hoyer J, Vasileiou G, Uebe S et al. Addition of triple negativity of breast cancer as an indicator for germline mutations in predisposing genes increases sensitivity of clinical selection criteria. BMC Cancer 2018; 18: 926

[16] Baselga J, Campone M, Piccart M et al. Everolimus in postmenopausal hormone-receptor-positive advanced breast cancer. N Engl J Med 2012; 366: 520-529

[17] Tesch H, Stoetzer O, Decker T et al. Efficacy and safety of everolimus plus exemestane in postmenopausal women with hormone receptor-positive, human epidermal growth factor receptor 2-negative locally advanced or metastatic breast cancer: Results of the single-arm, phase IIIB 4EVER trial. Int J Cancer 2019; 144: 877-885

[18] Banys-Paluchowski M, Krawczyk N, Paluchowski P. Cyclin-dependent kinase 4/6 inhibitors: what have we learnt across studies, therapy situations and substances. Curr Opin Obstet Gynecol 2019; 31: 56-66

[19] Turner NC, Slamon DJ, Ro J et al. Overall Survival with Palbociclib and Fulvestrant in Advanced Breast Cancer. N Engl J Med 2018; 379: 1926-1936

[20] Muller V, Nabieva N, Haberle L et al. Impact of disease progression on health-related quality of life in patients with metastatic breast cancer in the PRAEGNANT breast cancer registry. Breast 2018; 37: 154-160

[21] Rugo HS, Dieras V, Gelmon KA et al. Impact of palbociclib plus letrozole on patient-reported health-related quality of life: results from the PALOMA-2 trial. Ann Oncol 2018; 29: 888-894

[22] Huober ], Fasching PA, Taran FA et al. Factors associated with first line chemotherapy use in patients with hormone receptor positive, HER2 negative metastatic breast cancer - data from the PRAEGNANT breast cancer registry. Cancer Res 2018. doi:10.1158/1538-7445.SABCS1117P1153-1111-1107

[23] Hartkopf AD, Huober J, Volz B et al. Treatment landscape of advanced breast cancer patients with hormone receptor positive HER2 negative tumors - Data from the German PRAEGNANT breast cancer registry. Breast 2018; 37: 42-51

[24] Cancer Genome Atlas Network. Comprehensive molecular portraits of human breast tumours. Nature 2012; 490: 61-70

[25] Lux MP, Fasching PA, Schrauder MG et al. The PI3K Pathway: Background and Treatment Approaches. Breast Care (Basel) 2016; 11: 398-404

[26] O'Leary B, Cutts R], Liu Y et al. The Genetic Landscape and Clonal Evolution of Breast Cancer Resistance to Palbociclib plus Fulvestrant in the PALOMA-3 Trial. Cancer Discov 2018; 8: 1390-1403

[27] André F, Ciruelos E, Rubovszky G et al. Alpelisib (ALP) + fulvestrant (FUL) for advanced breast cancer $(A B C)$ : results of the Phase 3 SOLAR-1 trial. Proceedings of the ESMO Conference 2018. Ann Oncol 2018; Abstr. LBA3_PR

[28] Juric D, Ciruelos E, Rubovszky G et al. Alpelisib + fulvestrant for advanced breast cancer: Subgroup analyses from the phase III SOLAR-1 trial. San Antonio Breast Cancer Symposium 2018; Abstr. GS3-08

[29] Slamon DJ, Clark GM, Wong SG et al. Human breast cancer: correlation of relapse and survival with amplification of the HER-2/neu oncogene. Science 1987; 235: 177-182

[30] Slamon D, Eiermann W, Robert N et al. Adjuvant trastuzumab in HER2positive breast cancer. N Engl J Med 2011; 365: 1273-1283 
[31] Slamon D], Leyland-Jones B, Shak S et al. Use of chemotherapy plus a monoclonal antibody against HER2 for metastatic breast cancer that overexpresses HER2. N Engl J Med 2001; 344: 783-792

[32] Connell CM, Doherty G]. Activating HER2 mutations as emerging targets in multiple solid cancers. ESMO Open 2017; 2: e000279

[33] Konecny G, Pauletti G, Pegram M et al. Quantitative association between HER-2/neu and steroid hormone receptors in hormone receptor-positive primary breast cancer. J Natl Cancer Inst 2003; 95: 142-153

[34] Loibl S, von Minckwitz G, Schneeweiss A et al. PIK3CA mutations are associated with lower rates of pathologic complete response to anti-human epidermal growth factor receptor 2 (her2) therapy in primary HER2-overexpressing breast cancer. J Clin Oncol 2014; 32: 3212-3220

[35] Musolino A, Naldi N, Bortesi B et al. Immunoglobulin G fragment C receptor polymorphisms and clinical efficacy of trastuzumab-based therapy in patients with HER-2/neu-positive metastatic breast cancer. J Clin Oncol 2008; 26: 1789-1796

[36] Schettini F, Buono G, Cardalesi C et al. Hormone Receptor/Human Epidermal Growth Factor Receptor 2-positive breast cancer: Where we are now and where we are going. Cancer Treat Rev 2016; 46: 20-26

[37] Nuciforo P, Thyparambil S, Aura C et al. High HER2 protein levels correlate with increased survival in breast cancer patients treated with antiHER2 therapy. Mol Oncol 2016; 10: 138-147

[38] Swain SM, Kim SB, Cortes ] et al. Pertuzumab, trastuzumab, and docetaxel for HER2-positive metastatic breast cancer (CLEOPATRA study): overall survival results from a randomised, double-blind, placebo-controlled, phase 3 study. Lancet Oncol 2013; 14: 461-471

[39] Swain SM, Baselga J, Kim SB et al. Pertuzumab, trastuzumab, and docetaxel in HER2-positive metastatic breast cancer. N Engl J Med 2015; 372 . 724-734

[40] Baselga J, Cortes J, Kim SB et al. Pertuzumab plus trastuzumab plus docetaxel for metastatic breast cancer. N Engl J Med 2012; 366: 109-119

[41] Verma S, Miles D, Gianni L et al. Trastuzumab emtansine for HER2-positive advanced breast cancer. N Engl J Med 2012; 367: 1783-1791

[42] Wockel A, Festl J, Stuber T et al. Interdisciplinary Screening, Diagnosis, Therapy and Follow-up of Breast Cancer. Guideline of the DGGG and the DKG (S3-Level, AWMF Registry Number 032/045OL, December 2017) - Part 2 with Recommendations for the Therapy of Primary, Recurrent and Advanced Breast Cancer. Geburtsh Frauenheilk 2018; 78: 1056-1088

[43] Fasching PA, Brucker SY, Fehm TN et al. Biomarkers in Patients with Metastatic Breast Cancer and the PRAEGNANT Study Network. Geburtsh Frauenheilk 2015; 75: 41-50

[44] Lux MP, Nabieva N, Hartkopf AD et al. Therapy Landscape in Patients with Metastatic HER2-Positive Breast Cancer: Data from the PRAEGNANT Real-World Breast Cancer Registry. Cancers (Basel) 2018. doi:10.3390/ cancers 11010010

[45] Takegawa N, Nonagase Y, Yonesaka K et al. DS-8201a, a new HER2-targeting antibody-drug conjugate incorporating a novel DNA topoisomerase I inhibitor, overcomes HER2-positive gastric cancer T-DM1 resistance. Int J Cancer 2017; 141: 1682-1689

[46] Doi T, Shitara K, Naito Y et al. Safety, pharmacokinetics, and antitumour activity of trastuzumab deruxtecan (DS-8201), a HER2-targeting antibody-drug conjugate, in patients with advanced breast and gastric or gastro-oesophageal tumours: a phase 1 dose-escalation study. Lancet Oncol 2017; 18: 1512-1522

[47] Iwata H, Tamura K, Doi T et al. Trastuzumab deruxtecan (DS-8201a) in subjects with HER2-expressing solid tumors: Long-term results of a large phase 1 study with multiple expansion cohorts. J Clin Oncol 2018. doi:10.1200/JCO.2018.1236.1215_suppl.2501
[48] Powell CA, Camidge DR, Gemma A et al. Characterization, monitoring, and management of interstitial lung disease in patients with metastatic breast cancer: Analysis of data available from multiple studies of DS8201a, a HER2-targeted antibody drug conjugate with a topoisomerase I inhibitor payload. San Antonio Breast Cancer Symposium 2018; Abstr. P6-17-06

[49] Schneeweiss A, Lux MP, Hartkopf AD et al. Progression free survival (PFS) and overall survival (OS) of patients treated with trastuzumab emtansine (T-DM1) after previous treatment with pertuzumab in patients with advanced breast cancer (NCT02338167). San Antonio Breast Cancer Symposium 2018; Abstr. P6-17-22

[50] Witzel I, Laakmann E, Weide R et al. Treatment and outcomes of patients in the Brain Metastases in Breast Cancer Network Registry. Eur J Cancer 2018; 102: 1-9

[51] Sperduto PW, Kased N, Roberge D et al. Summary report on the graded prognostic assessment: an accurate and facile diagnosis-specific tool to estimate survival for patients with brain metastases. J Clin Oncol 2012; 30: $419-425$

[52] Witzel ID, Riecke K, Laakmann E et al. Validation of different prognostic scores in breast cancer patients with brain metastases of the BMBC registry (GBG-79). San Antonio Breast Cancer Symposium 2018; Abstr. P4-08-26

[53] Tolaney SM, Sahebjam S, Le Rhun E et al. A phase 2 study of abemaciclib in patients with leptomeningeal metastases secondary to HR+, HER2breast cancer. San Antonio Breast Cancer Symposium 2018; Abstr. P119-07

[54] Braun S, Vogl FD, Naume B et al. A pooled analysis of bone marrow micrometastasis in breast cancer. N Engl J Med 2005; 353: 793-802

[55] Trapp E, Janni W, Schindlbeck C et al. Presence of Circulating Tumor Cells in High-Risk Early Breast Cancer During Follow-Up and Prognosis. J Natl Cancer Inst 2018. doi:10.1093/jnci/djy152

[56] Huebner H, Fasching PA, Gumbrecht W et al. Filtration based assessment of CTCs and CellSearch(R) based assessment are both powerful predictors of prognosis for metastatic breast cancer patients. BMC Cancer 2018; 18: 204

[57] Polasik A, Tzschaschel M, Schochter F et al. Circulating Tumour Cells, Circulating Tumour DNA and Circulating MicroRNA in Metastatic Breast Carcinoma - What is the Role of Liquid Biopsy in Breast Cancer? Geburtsh Frauenheilk 2017; 77: 1291-1298

[58] Janni W], Rack B, Terstappen LW et al. Pooled Analysis of the Prognostic Relevance of Circulating Tumor Cells in Primary Breast Cancer. Clin Cancer Res 2016; 22: 2583-2593

[59] Janni W, Rack B, Fasching P et al. Persistence of circulating tumor cells in high risk early breast cancer patients during follow-up care suggests poor prognosis - Results from the adjuvant SUCCESS A trial. Cancer Res 2016. doi:10.1158/1538-7445.SABCS15-S2-03

[60] Rack B, Schindlbeck C, Juckstock J et al. Circulating tumor cells predict survival in early average-to-high risk breast cancer patients. J Natl Cancer Inst 2014. doi:10.1093/jnci/dju066

[61] Muller V, Riethdorf S, Rack B et al. Prognostic impact of circulating tumor cells assessed with the CellSearch System and AdnaTest Breast in metastatic breast cancer patients: the DETECT study. Breast Cancer Res 2012; 14: R118

[62] Hosseini H, Obradovic MM, Hoffmann M et al. Early dissemination seeds metastasis in breast cancer. Nature 2016. doi:10.1038/nature20785

[63] Hartkopf AD, Brucker SY, Taran FA et al. International pooled analysis of the prognostic impact of disseminated tumor cells from the bone marrow in early breast cancer: Results from the PADDY study. San Antonio Breast Cancer Symposium 2018; Abstr. GS5-07

[64] Bidard FC, Peeters DJ, Fehm T et al. Clinical validity of circulating tumour cells in patients with metastatic breast cancer: a pooled analysis of individual patient data. Lancet Oncol 2014; 15: 406-414 
[65] Bidard FC, Jacot W, Dureau S et al. Clinical utility of circulating tumor cell count as a tool to chose between first line hormone therapy and chemotherapy for ER+ HER2- metastatic breast cancer: Results of the phase III STIC CTC trial. San Antonio Breast Cancer Symposium 2018; Abstr. GS307

[66] Ganz PA, Yip CH, Gralow JR et al. Supportive care after curative treatment for breast cancer (survivorship care): resource allocations in lowand middle-income countries. A Breast Health Global Initiative 2013 consensus statement. Breast 2013; 22: 606-615

[67] Cardoso F, Bese N, Distelhorst SR et al. Supportive care during treatment for breast cancer: resource allocations in low- and middle-income countries. A Breast Health Global Initiative 2013 consensus statement. Breast 2013; 22: 593-605

[68] Harrison JD, Young JM, Price MA et al. What are the unmet supportive care needs of people with cancer? A systematic review. Support Care Cancer 2009; 17: 1117-1128

[69] Beesley VL, Alemayehu C, Webb PM. A systematic literature review of the prevalence of and risk factors for supportive care needs among women with gynaecological cancer and their caregivers. Support Care Cancer 2018; 26: 701-710

[70] Distelhorst SR, Cleary JF, Ganz PA et al. Optimisation of the continuum of supportive and palliative care for patients with breast cancer in low-income and middle-income countries: executive summary of the Breast Health Global Initiative, 2014. Lancet Oncol 2015; 16: e137-e147

[71] Aapro MS, Bohlius ], Cameron DA et al. 2010 update of EORTC guidelines for the use of granulocyte-colony stimulating factor to reduce the incidence of chemotherapy-induced febrile neutropenia in adult patients with lymphoproliferative disorders and solid tumours. Eur J Cancer 2011; 47: 8-32

[72] Olver I, Clark-Snow RA, Ballatori E et al. Guidelines for the control of nausea and vomiting with chemotherapy of low or minimal emetic potential. Support Care Cancer 2011; 19 (Suppl. 1): S33-S36

[73] Jordan K, Roila F, Molassiotis A et al. Antiemetics in children receiving chemotherapy. MASCC/ESMO guideline update 2009. Support Care Cancer 2011; 19 (Suppl. 1): S37-S42

[74] Jordan K, Aapro M, Kaasa S et al. European Society for Medical Oncology (ESMO) position paper on supportive and palliative care. Ann Oncol 2018; 29: 36-43

[75] Hesketh PJ, Kris MG, Basch E et al. Antiemetics: American Society of Clinical Oncology Clinical Practice Guideline Update. J Clin Oncol 2017; 35: 3240-3261

[76] Feyer PC, Maranzano E, Molassiotis A et al. Radiotherapy-induced nausea and vomiting (RINV): MASCC/ESMO guideline for antiemetics in radiotherapy: update 2009. Support Care Cancer 2011; 19 (Suppl. 1): S5-S14

[77] de Wit M, Ortner P, Lipp HP et al. Management of cytotoxic extravasation - ASORS expert opinion for diagnosis, prevention and treatment. Onkologie 2013; 36: 127-135

[78] Bossi P, Antonuzzo A, Cherny NI et al. Diarrhoea in adult cancer patients: ESMO Clinical Practice Guidelines. Ann Oncol 2018; 29: iv126-iv142

[79] Slamon DJ, Eiermann W, Robert NJ et al. Ten year follow-up of BCIRG-006 comparing doxorubicin plus cyclophosphamide followed by docetaxel (AC -> T) with doxorubicin plus cyclophosphamide followed by docetaxel and trastuzumab (AC $>\mathrm{TH}$ ) with docetaxel, carboplatin and trastuzumab (TCH) in HER2+early breast cancer. Cancer Res 2016. doi:10.1158/1538-7445.SABCS1115-S1155-1104

[80] Janni W, Nitz U, Rack BK et al. Pooled analysis of two randomized phase III trials (PlanB/SuccessC) comparing six cycles of docetaxel and cyclophosphamide to sequential anthracycline taxane chemotherapy in patients with intermediate and high risk HER2-negative early breast cancer ( $n=5,923)$. J Clin Oncol 2018; 36: 522. doi:510.1200/ JCO.2018.1236.1215_suppl.1522
[81] Munster P, Krischer ], Tamura R et al. A randomized community-based trial of an angiotensin converting enzyme inhibitor, lisinopril or a beta blocker, carvedilol for the prevention of cardiotoxicity in patients with early stage HER2-positive breast cancer receiving adjuvant trastuzumab. San Antonio Breast Cancer Symposium 2018; Abstr. GS5-01

[82] Nabieva N, Kellner S, Fehm T et al. Influence of patient and tumor characteristics on early therapy persistence with letrozole in postmenopausal women with early breast cancer: results of the prospective Evaluate-TM study with 3941 patients. Ann Oncol 2018; 29: 186-192

[83] Nabieva N, Fehm T, Haberle L et al. Influence of side-effects on early therapy persistence with letrozole in post-menopausal patients with early breast cancer: Results of the prospective EvAluate-TM study. Eur J Cancer 2018; 96: 82-90

[84] Chirgwin JH, Giobbie-Hurder A, Coates AS et al. Treatment Adherence and Its Impact on Disease-Free Survival in the Breast International Group 1-98 Trial of Tamoxifen and Letrozole, Alone and in Sequence. J Clin Oncol 2016; 34: 2452-2459

[85] Buser KS, Joss RA, Piquet D et al. Oral ondansetron in the prophylaxis of nausea and vomiting induced by cyclophosphamide, methotrexate and 5-fluorouracil (CMF) in women with breast cancer. Results of a prospective, randomized, double-blind, placebo-controlled study. Ann Oncol 1993; 4: 475-479

[86] Schilling J, Hielscher C, Hanusch C et al. Efficacy of NEPA as antiemetic prophylaxis in breast cancer patients receiving highly or moderately emetogenic chemotherapy - Interim results of a German prospective, noninterventional study. San Antonio Breast Cancer Symposium 2018; Abstr. P1-11-10

[87] Fasching PA, Kollmannsberger B, Strissel PL et al. Polymorphisms in the novel serotonin receptor subunit gene HTR3C show different risks for acute chemotherapy-induced vomiting after anthracycline chemotherapy. J Cancer Res Clin Oncol 2008; 134: 1079-1086

[88] Hammer C, Fasching PA, Loehberg CR et al. Polymorphism in HTR3D shows different risks for acute chemotherapy-induced vomiting after anthracycline chemotherapy. Pharmacogenomics 2010; 11: 943-950

[89] Kollmannsberger B, Lohberg C, Stohlmacher ] et al. Prediction of emesis by polymorphisms in the serotonin receptor subunit gene HTR3C - Rationale for an international prospective pharmacogenomics study Emesis 2 - CINV. Onkologie 2008; 31: 27-28

[90] Lopez-Morales P, Flores-Funes D, Sanchez-Migallon EG et al. Genetic Factors Associated with Postoperative Nausea and Vomiting: a Systematic Review. J Gastrointest Surg 2018; 22: 1645-1651

[91] Sparano JA, Gray RJ, Makower DF et al. Adjuvant Chemotherapy Guided by a 21-Gene Expression Assay in Breast Cancer. N Engl J Med 2018; 379 : 111-121

[92] Wagner LI, Gray RJ, Garcia S et al. Symptoms and health-related quality of life on endocrine therapy alone (E) versus chemoendocrine therapy (C $+\mathrm{E})$ : TAILORx patient-reported outcomes results. San Antonio Breast Cancer Symposium 2018; Abstr. GS6-03

[93] Janni W, Rack BK, Friedl TW et al. Lifestyle Intervention and Effect on Disease-free Survival in Early Breast Cancer Pts: Interim Analysis from the Randomized SUCCESS C Study. San Antonio Breast Cancer Symposium 2018; Abstr. GS5-03

[94] Thune I, Husoy A, Frydenberg $\mathrm{H}$ et al. Cardiovascular function and the effect of exercise training during adjuvant breast cancer treatment. Results from The EBBA-II trial. San Antonio Breast Cancer Symposium 2018; Abstr. GS5-02

[95] Hurria A, Magnuson A, Gross CP et al. Development and validation of a chemotherapy toxicity (Chemo Tox) risk score for older patients (Pts) with breast cancer $(\mathrm{BC})$ receiving adjuvant/neoadjuvant treatment (Adjuvant Tx): A R01 and BCRF funded prospective multicenter study. San Antonio Breast Cancer Symposium 2018; Abstr. GS6-04

[96] Schmidt M, Fasching PA, Beckmann MW et al. Biomarkers in Breast Cancer - An Update. Geburtsh Frauenheilk 2012; 72: 819-832 
[97] Andre F, Filleron T, $\mathrm{Ng} \mathrm{C}$ et al. Genomic characterisation of metastatic breast cancer. San Antonio Breast Cancer Symposium 2018; Abstr. GS108

[98] Andre F, Bachelot T, Commo F et al. Comparative genomic hybridisation array and DNA sequencing to direct treatment of metastatic breast cancer: a multicentre, prospective trial (SAFIR01/UNICANCER). Lancet Oncol 2014; 15: 267-274

[99] Le Tourneau C, Delord JP, Goncalves A et al. Molecularly targeted therapy based on tumour molecular profiling versus conventional therapy for advanced cancer (SHIVA): a multicentre, open-label, proof-of-concept, randomised, controlled phase 2 trial. Lancet Oncol 2015; 16: 1324-1334

[100] Massard C, Michiels S, Ferte C et al. High-Throughput Genomics and Clinical Outcome in Hard-to-Treat Advanced Cancers: Results of the MOSCATO 01 Trial. Cancer Discov 2017; 7: 586-595
[101] Wunderle M, Olmes G, Nabieva N et al. Risk, Prediction and Prevention of Hereditary Breast Cancer - Large-Scale Genomic Studies in Times of Big and Smart Data. Geburtsh Frauenheilk 2018; 78: 481-492

[102] Shimelis H, LaDuca H, Hu C et al. Triple-Negative Breast Cancer Risk Genes Identified by Multigene Hereditary Cancer Panel Testing. J Natl Cancer Inst 2018. doi:10.1093/jnci/djy106

[103] Hein A, Gass P, Walter CB et al. Computerized patient identification for the EMBRACA clinical trial using real-time data from the PRAEGNANT network for metastatic breast cancer patients. Breast Cancer Res Treat 2016; 158: 59-65

[104] Tresp V, Overhage JM, Bundschus M et al. Going Digital: A Survey on Digitalization and Large-Scale Data Analytics in Healthcare. P IEEE 2016; 104: 2180-2206 